\title{
Sweepouts of amalgamated 3-manifolds
}

\author{
DAVID BACHMAN \\ SAUL SCHLEIMER \\ ERIC SEDGWICK
}

\begin{abstract}
We show that if two 3-manifolds with toroidal boundary are glued via a "sufficiently complicated" map then every Heegaard splitting of the resulting 3-manifold is weakly reducible. Additionally, suppose $X \cup_{F} Y$ is a manifold obtained by gluing $X$ and $Y$, two connected small manifolds with incompressible boundary, along a closed surface $F$. Then the following inequality on genera is obtained:

$$
g\left(X \cup_{F} Y\right) \geq \frac{1}{2}(g(X)+g(Y)-2 g(F)) .
$$

Both results follow from a new technique to simplify the intersection between an incompressible surface and a strongly irreducible Heegaard splitting.
\end{abstract}

57N10, 57M99; 57M27

\section{Introduction}

It is a consequence of the Haken Lemma [4] and the Uniqueness of Prime Decompositions, Kneser [8], that Heegaard genus is well behaved under connected sum. In particular, 3-manifold genus is additive:

$$
g(X \# Y)=g(X)+g(Y)
$$

Here we discuss the Heegaard splittings of a manifold obtained by gluing together manifolds along boundary components of higher genus.

To this end let $X$ and $Y$ be 3-manifolds with incompressible boundary homeomorphic to a connected surface $F$. It is not difficult to show that if $H_{X}$ and $H_{Y}$ are Heegaard surfaces in $X$ and $Y$ then we can amalgamate these splittings to obtain a Heegaard surface in $X \cup_{F} Y$ with genus equal to $g\left(H_{X}\right)+g\left(H_{Y}\right)-g(F)$ (see, for example, Schultens [14]). Letting $g(X), g(Y)$, and $g\left(X \cup_{F} Y\right)$ denote the minimal genus among all Heegaard surfaces in the respective 3-manifolds, we find:

$$
g\left(X \cup_{F} Y\right) \leq g(X)+g(Y)-g(F)
$$


Bounds in the other direction are harder to obtain. When $F \cong S^{2}$ it follows from the Haken Lemma [4] that the above inequality may be replaced by an equality. In Section 4 we examine the case where $F$ is a torus. We assume here that the map which identifies $\partial X$ to $\partial Y$ is "sufficiently complicated," in a sense to be made precise in Section 4.

Theorem 4.1 Suppose that $X$ and $Y$ are knot manifolds and $\varphi: \partial X \rightarrow \partial Y$ is a sufficiently complicated homeomorphism. Then the manifold $M(\varphi)=X \cup_{\varphi} Y$ has no strongly irreducible Heegaard splittings.

In particular it follows from this result that every Heegaard splitting of $X \cup_{F} Y$ is an amalgamation of splittings of $X$ and $Y$. In this situation Inequality (1) becomes an equality.

In the case where the genus of $F$ is at least two there is the following result of Lackenby [9]:

Theorem Let $X$ and $Y$ be simple 3-manifolds, and let $h: \partial X \rightarrow F$ and $h^{\prime}: F \rightarrow \partial Y$ be homeomorphisms with some connected surface $F$ of genus at least two. Let $\psi: F \rightarrow F$ be a psuedo-Anosov homeomorphism. Then, provided $|n|$ is sufficiently large,

$$
g\left(X \cup_{h^{\prime} \psi^{n} h} Y\right)=g(X)+g(Y)-g(F) .
$$

Furthermore, any minimal genus Heegaard splitting for $X \cup_{h^{\prime} \psi^{n} h} Y$ is obtained from splittings of $X$ and $Y$ by amalgamation, and hence is weakly reducible.

If $\psi$ fails to be "sufficiently complicated" then there is no hope of an exact equality, as in the previous theorem. Previous known lower bounds were obtained by Johannson [7] when $X$ and $Y$ are simple

$$
g\left(X \cup_{F} Y\right) \geq \frac{1}{5}(g(X)+g(Y)-2 g(F)) .
$$

Schultens has generalized this result to allow essential annuli [13].

By assuming the component manifolds $X$ and $Y$ are small we get a new bound. The following statement is one case of Theorem 5.1:

Theorem 5.1' Suppose $X$ and $Y$ are compact, orientable, connected, small 3-manifolds with incompressible boundary homeomorphic to a surface $F$. Then

$$
g\left(X \cup_{F} Y\right) \geq \frac{1}{2}(g(X)+g(Y)-2 g(F)) .
$$

Algebraic 83 Geometric Topology, Volume 6 (2006) 
Both of our results follow from showing that a strongly irreducible Heegaard surface $H$ can be isotoped to meet the gluing surface $F$ in a particularly nice fashion. Often in these types of arguments one simplifies the intersection by making every loop of $H \cap F$ essential in both surfaces. In this paper, rather than focusing on the intersection set $H \cap F$, we focus on the complimentary pieces $H \backslash N(F)$. Our result is that $H$ and $F$ may always be arranged so that almost every component $H^{\prime}$ of $H \backslash N(F)$ is incompressible. On such a component every loop which is essential in $H^{\prime}$ is essential in $M \backslash N(F)$. There is at most one component $H^{\prime \prime}$ which is compressible. In this case we find that $H^{\prime \prime}$ is strongly irreducible, in the sense that every essential loop which bounds a disk on one side meets every essential loop bounding a disk on the other. See Lemma 3.3.

\section{Definitions}

In this section we give some of the standard definitions that will be used throughout paper.

\subsection{Essential loops, arcs, and surfaces}

A loop $\gamma$ embedded in the interior of a compact, orientable surface $F$ is called essential if it does not bound a disk in $F$. If $F$ is embedded in a 3-manifold, $M$, a compressing disk for $F$ is a disk, $D \subset M$, such that $F \cap D=\partial D$, and such that $\partial D$ is essential on $F$. If we identify a thickening of $D$ in $M \backslash N(F)$ with $D \times I$ then to compress $F$ along $D$ is to remove $(\partial D) \times I$ from $F$ and replace it with $D \times \partial I$.

A properly embedded arc $\alpha$ on $F$ is essential if there is no subarc $\beta$ of $\partial F$ such that $\alpha \cup \beta$ is the boundary of a subdisk of $F$. If $F$ is properly embedded in a 3manifold, $M$, a boundary-compressing disk is a disk, $D$, such that $\partial D=\alpha \cup \beta$, where $F \cap D=\alpha$ is an essential arc on $F$ and $D \cap \partial M=\beta$. If we identify a thickening of $D$ in $M \backslash N(F)$ with $D \times I$ then to boundary-compress $F$ along $D$ is to remove $\alpha \times I$ from $F$ and replace it with $D \times \partial I$.

A properly embedded surface is incompressible if there are no compressing disks for it. A properly embedded, separating surface is strongly irreducible if there are compressing disks for it on both sides, and each compressing disk on one side meets each compressing disk on the other side.

A compact, orientable 3-manifold is said to be irreducible if every embedded 2sphere bounds a 3-ball. A 3-manifold is said to be small if it is irreducible and every incompressible surface is parallel to a boundary component. 


\subsection{Heegaard and generalized Heegaard Splittings.}

A compression body is a 3-manifold $C$ constructed in one of two different ways. The first way is to begin with a collection of zero-handles and attach one-handles to their boundaries, resulting in a manifold that may or may not be connected. In this case we say the spine of $C$ is a 1-complex $\Sigma$ in $C$ such that $C$ is homeomorphic to a thickening of $\Sigma$. We set $\partial_{-} C=\varnothing$ and $\partial_{+} C=\partial C$.

The second way to construct a compression body is to begin with a closed (possibly disconnected) orientable surface $F$ with no sphere components, and let $C$ be the manifold obtained by attaching one-handles to the surface $F \times\{1\} \subset F \times I$. In this case we say $\partial_{-} C=F \times\{0\}$ and $\partial_{+} C=\partial C \backslash \partial_{-} C$. The spine $\Sigma$ is then the union of $\partial_{-} C$ and a collection of arcs which are properly embedded in $C$, such that $C$ is a thickening of $\Sigma$.

A surface, $H$, in a 3-manifold, $M$, is a Heegaard surface for $M$ if $H$ separates $M$ into two compression bodies, $V$ and $W$, such that $H=\partial_{+} V=\partial_{+} W$.

A generalized Heegaard splitting of a 3-manifold $M$, Scharlemann-Thompson [12], is a sequence $\left\{H_{i}\right\}_{i=0}^{2 n}$ of pairwise disjoint, closed surfaces in $M$ such that

- $\partial M=H_{0} \cup H_{2 n}$ (if $\partial M=\varnothing$ then $H_{0}=H_{2 n}=\varnothing$ ) and

- for each odd $i$, the surface $H_{i}$ is a Heegaard splitting of the submanifold cobounded by $H_{i-1}$ and $H_{i+1}$.

We will call the set of surfaces with even index thin levels and the set with odd index thick levels.

Generalized Heegaard splittings are associated to handle structures in the following way. Given a generalized Heegaard splitting $\left\{H_{i}\right\}_{i=0}^{n}$ there is a sequence of submanifolds $\left\{M_{i}\right\}$ of $M$ as follows:

- $M_{0}$ is a union of zero-handles and 1-handles.

- For odd $i$ between 1 and $n, M_{i}$ is obtained from $M_{i-1}$ by attaching onehandles.

- For even $i$ between 2 and $n-1, M_{i}$ is obtained from $M_{i-1}$ by attaching two-handles.

- $M_{n}=M$ is obtained from $M_{n-1}$ by attaching two-handles and three-handles.

Conversely, given a handle structure for $M$ there is an associated generalized Heegaard splitting as above.

Algebraic 83 Geometric Topology, Volume 6 (2006) 
Suppose $H_{X}$ and $H_{Y}$ are Heegaard surfaces in 3-manifolds $X$ and $Y$. Suppose further that the boundaries of both $X$ and $Y$ are homeomorphic to a surface $F$. Then $\left\{\varnothing, H_{X}, F, H_{Y}, \varnothing\right\}$ is a generalized Heegaard splitting of $X \cup_{F} Y$. We may now choose a handle structure associated to this generalized Heegaard splitting, and re-arrange it so that handles are added in order of increasing index. The generalized Heegaard splitting associated to this new handle structure will be of the form $\{\varnothing, H, \varnothing\}$, where $H$ is a Heegaard surface in $X \cup_{F} Y$. In this case the Heegaard surface $H$ is the amalgamation of $H_{X}$ and $H_{Y}$, as defined by Schultens [14].

\subsection{Normal and almost normal surfaces.}

A normal disk in a tetrahedron is a triangle or a quadrilateral, as in Figure 1. Let $X$ be a 3-manifold equipped with a psuedo-triangulation. That is, $X$ is expressed as a collection of tetrahedra, together with face pairings.
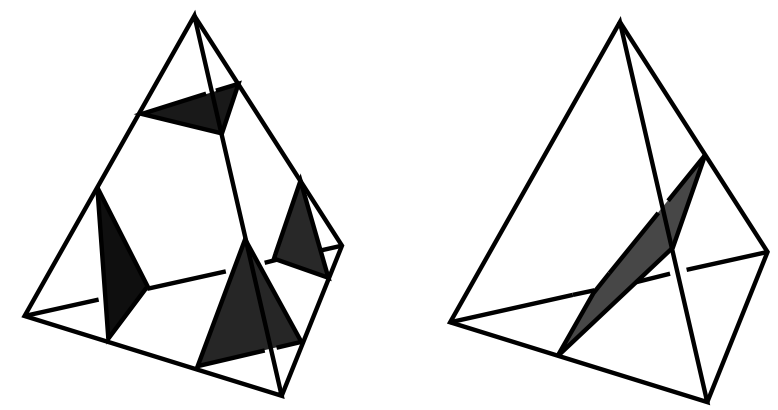

Figure 1: Normal disks

A properly embedded surface in $X$ is normal if it intersects every tetrahedron in a collection of triangles and quadrilaterals. Normal surfaces were first introduced by Kneser [8], and later used to solve several important problems by Haken [3].

A properly embedded surface in $X$ is almost normal if it is normal everywhere, with the exception of exactly one piece in one tetrahedron. The exceptional piece can either be an octagon, two normal disks connected by an unknotted tube, or two normal disks connected by a band along $\partial X$ (see Figure 2). In the closed case, almost normal surfaces were introduced by Rubinstein [10]. They were later generalized to surfaces with non-empty boundary by the first author [1].

\section{Labelling sweepouts}

In this section we prove the technical lemmas on which Sections 4 and 5 rely. 

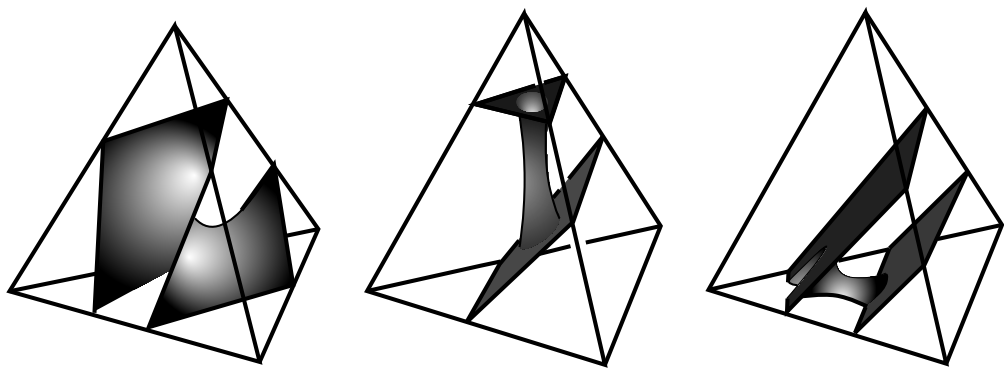

Figure 2: Exceptional disks in an almost normal surface

Lemma 3.1 (Scharlemann [11]) Let $H$ be a strongly irreducible Heegaard surface, and $\gamma$ be an essential curve on $H$. Suppose $\gamma$ bounds a disk $D \subset M$ such that $D$ is transverse to $H$. Then $\gamma$ bounds a compressing disk for $H$.

Definition 3.2 Two surfaces $H$ and $F$ embedded in a 3-manifold are almost transverse if they have exactly one non-transverse intersection point, and it is a saddle point.

Lemma 3.3 Let $M$ be a compact, irreducible, orientable 3-manifold with $\partial M$ incompressible, if non-empty. Suppose $M=V \cup_{H} W$, where $H$ is a strongly irreducible Heegaard surface. Suppose further that $M$ contains an incompressible, orientable, closed, non-boundary parallel surface $F$. Then either

- $H$ may be isotoped to be transverse to $F$, with every component of $H \backslash N(F)$ incompressible in the respective submanifold of $M \backslash N(F)$,

- $H$ may be isotoped to be transverse to $F$, with every component of $H \backslash N(F)$ incompressible in the respective submanifold of $M \backslash N(F)$ except for exactly one strongly irreducible component, or

- $H$ may be isotoped to be almost transverse to $F$, with every component of $H \backslash N(F)$ incompressible in the respective submanifold of $M \backslash N(F)$.

\section{Remarks 3.4}

(1) After applying the lemma every loop of $H \cap F$ must be essential on both surfaces. Otherwise there is such a loop that is inessential on $F$ and essential on $H$. This loop, after a small isotopy, bounds a compressing disk $D$ for a component $H^{\prime}$ of $H \backslash N(F)$. By the lemma, $H^{\prime}$ must then be strongly irreducible. But $D$ is disjoint from every compressing disk for $H^{\prime}$ on the opposite side, a contradiction. 
(2) In the case where $F \cong \mathbb{T}^{2}$ it will follow from the proof that $H$ may actually be isotoped to be transverse to $F$. Here, only conclusions one or two of the lemma occur.

Proof of Lemma 3.3 Choose spines $\Sigma_{V}$ of $V$ and $\Sigma_{W}$ of $W$.

Claim 3.5 The surface $F$ meets both $\Sigma_{V}$ and $\Sigma_{W}$.

Proof Suppose $F \cap \Sigma_{V}=\varnothing$. Then $F$ lies in a compression body homeomorphic to $W$. As the only incompressible surfaces in $W$ are components of $\partial_{-} W$, we conclude that $F$ is boundary parallel in $M$. This violates the hypotheses of Lemma 3.3.

Fix a sweepout of $M:$ a continuous map $\Phi: H \times I \rightarrow M$ such that

- $H(0)=\Sigma_{V}$,

- $H(1)=\Sigma_{W}$, and

- the restriction of $\Phi$ to $H \times(0,1)$ is a smooth homeomorphism onto the complement of $\Sigma_{V} \cup \Sigma_{W}$.

Here $H(t)=\Phi(H \times t)$. The map $\Phi$ is a sweepout of $M$. (Note that this is a slightly different definition than the one introduced by Rubinstein). Let $V(t)$ and $W(t)$ denote the compression bodies bounded by $H(t)$ (where $\Sigma_{V} \subset V(t)$ ).

The sweepout $\Phi$ induces a height function $h: F \rightarrow I$ as follows. Define $h(x)=t$ if $x \in \Phi(H, t)$. Perturb $F$ so that $h$ is Morse on $F \backslash\left(\Sigma_{V} \cup \Sigma_{W}\right)$. Let $\left\{t_{i}\right\}_{i=0}^{n}$ denote the set of critical values of $h$. It follows from Claim 3.5 that $t_{0}=0$ and $t_{n}=1$. We now label each subinterval $\left(t_{i}, t_{i+1}\right)$ with the letters $\mathbb{V}$ and/or $\mathbb{W}$ by the following scheme. If, for some $t \in\left(t_{i}, t_{i+1}\right)$, there is a compressing disk for $H(t)$ in $V(t)$ with boundary disjoint from $F$ then label this subinterval with the letter $\mathbb{V}$. See Figure 3. Similarly, if there is a compressing disk in $W(t)$ with boundary disjoint from $F$ then label with the letter $\mathbb{W}$.

Claim 3.6 If the subinterval $\left(t_{i}, t_{i+1}\right)$ is unlabelled then the first conclusion of Lemma 3.3 follows.

Proof Suppose $t \in\left(t_{i}, t_{i+1}\right)$. First, we claim that all curves of $H(t) \cap F$ are essential on both or inessential on both. If not then, as $F$ is incompressible, there is a loop $\delta \subset H(t) \cap F$ that is inessential on $F$ but essential on $H(t)$. The loop $\delta$ bounds a disk $D \subset F$. Thus the hypotheses of Lemma 3.1 are satisfied. It follows that $\delta$ bounds a compressing disk in $V(t)$ or in $W(t)$. Finally, $\delta$ may be isotoped inside of $H(t)$ by a 


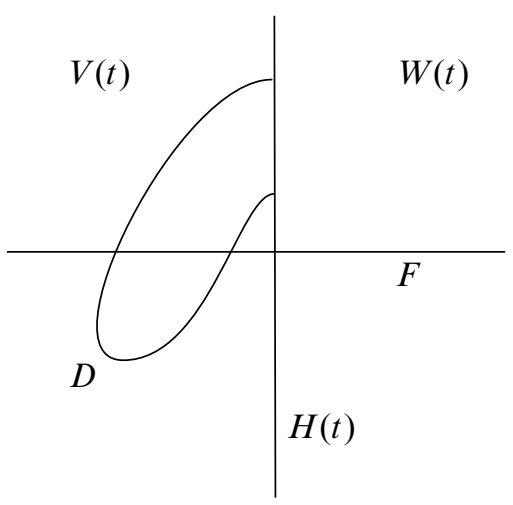

Figure 3: If $D$ is a compressing disk for $H(t)$ in $V(t)$ with boundary disjoint from $F$ then the interval containing $t$ would get the label $\mathbb{V}$.

small pushout move to be disjoint from $F$. This violates the assumption that $\left(t_{i}, t_{i+1}\right)$ is unlabelled. We deduce that all curves of $H(t) \cap F$ are essential or inessential on both.

As $M$ is irreducible we may isotope $H(t)$ to remove those loops of $H(t) \cap F$ which are inessential on both surfaces, without affecting those loops of $H(t) \cap F$ which were essential on both. We now claim that after such an isotopy any essential loop of $H(t) \backslash N(F)$ is essential on $H(t)$. We prove the contrapositive: Suppose $E \subset H(t)$ is an embedded disk with $\partial E \cap F=\varnothing$. All curves of $E \cap F$ are inessential on both surfaces. Isotope $E$ rel boundary to make $E \cap F=\varnothing$. We conclude $E \subset M \backslash N(F)$, and hence $\partial E$ is inessential on $H(t) \backslash N(F)$.

Finally, we claim that the components of $H(t) \backslash N(F)$ are incompressible in the respective submanifolds of $M \backslash N(F)$. Suppose $H^{\prime}$ is a compressible component. Then there is an essential loop $\gamma \subset H^{\prime}$ which bounds a compressing disk for $H^{\prime}$. By the preceding remarks $\gamma$ is essential on $H(t)$ as well. By Lemma 3.1 the loop $\gamma$ bounds a compressing disk for $H(t)$, which must be in $V(t)$ or $W(t)$. This now contradicts the fact that $\left(t_{i}, t_{i+1}\right)$ is unlabelled.

Claim 3.7 If the subinterval $\left(t_{i}, t_{i+1}\right)$ has both of the labels $\mathbb{V}$ and $\mathbb{W}$ then the second conclusion of Lemma 3.3 follows.

Proof Suppose $t \in\left(t_{i}, t_{i+1}\right)$. We begin as in the proof of Claim 3.6 by asserting that all curves of $H(t) \cap F$ are either inessential or essential on both. If not, then as above there is a loop $\delta \subset H(t) \cap F$ which bounds a compressing disk for $H(t)$. Suppose $\delta$ bounds a compressing disk in $V(t)$. (The other case is similar.) Since $\left(t_{i}, t_{i+1}\right)$ has 
the label $\mathbb{W}$ there is a loop $\gamma$ on some component of $H(t) \backslash N(F)$ which bounds a disk in $W(t)$. But then $\delta \cap \gamma=\varnothing$ contradicts the strong irreducibility of $H$.

As in the proof of Claim 3.6 it now follows that we may isotope $H(t)$, preserving the set of loops of $H(t) \cap F$ which are essential on both, so that any loop which is essential on $H(t) \backslash N(F)$ is also essential on $H(t)$.

Let $H^{\prime}$ be a component of $H(t) \backslash N(F)$ which contains a loop $\gamma$ bounding a compressing disk for $H(t)$ in $W(t)$. By strong irreducibility of $H(t)$ any essential loop of $H(t) \backslash N(F)$ which bounds a compressing disk in $V(t)$ must meet $\gamma$, and hence must also lie in $H^{\prime}$. Furthermore, since the subinterval $\left(t_{i}, t_{i+1}\right)$ has the label $\mathbb{V}$, there is at least one such loop $\rho$. By identical reasoning we conclude that any essential loop of $H(t) \backslash N(F)$ which bounds a compressing disk in $W(t)$ must meet $\rho$, and hence must also be on $H^{\prime}$. We conclude that there are no loops on any other component of $H(t) \backslash N(F)$ which bound compressing disks. Hence all components of $(H(t) \backslash N(F)) \backslash H^{\prime}$ are incompressible in the respective submanifolds of $M \backslash N(F)$. Furthermore, the strong irreducibility of $H^{\prime}$ follows from the existence of the $\mathbb{V}$ and W labels and strong irreducibility of $H(t)$.

Claim 3.8 If the labelling of $\left(t_{i-1}, t_{i}\right)$ is different from that of $\left(t_{i}, t_{i+1}\right)$ then the critical value $t_{i}$ corresponds to a saddle tangency between $H\left(t_{i}\right)$ and $F$.

Claim 3.9 The subinterval $\left(0, t_{1}\right)$ is labelled $\mathbb{V}$ and the subinterval $\left(t_{n-1}, 1\right)$ is labelled W.

Proof For sufficiently small $\epsilon$ the surface $H(\epsilon)$ looks like the frontier of a neighborhood of $\Sigma_{V}$. By Claim 3.5 the surface $F$ meets $\Sigma_{V}$. Hence, $F$ contains small compressions for $H(\epsilon)$ in $V(\epsilon)$. We can push these compressions off $F$, giving compressions with boundary on a component of $H(\epsilon) \backslash N(F)$ in $V(\epsilon)$. Hence, the label of $\left(0, t_{1}\right)$ is $\mathbb{V}$. A symmetric argument completes the proof of the claim.

Following Claims 3.6 and 3.7 we now assume that every subinterval has a label. Furthermore, we assume that every subinterval has exactly one label: either $\mathbb{V}$ or $\mathbb{W}$, but not both. It then follows from Claim 3.9 that there is some first critical value $t_{i}$ where the labelling changes from $\mathbb{V}$ to $\mathbb{W}$. By Claim 3.8 this critical value must correspond to a saddle tangency.

Claim 3.10 There is a surface $H_{0}$, isotopic to $H\left(t_{i}\right)$, such that all components of $H_{0} \backslash N(F)$ are incompressible. 
Proof First, we claim that every component of $H\left(t_{i}\right) \cap F$ which is a loop is either essential or inessential on both surfaces. If not, then as in the proof of Claim 3.6 there is a loop component $\delta$ of $H\left(t_{i}\right) \cap F$ which bounds a compressing disk for $H\left(t_{i}\right)$. Assume that the compressing disk bounded by $\delta$ lies in $W\left(t_{i}\right)$, as the other case is similar. Pushing $\delta$ off of $F$ along $H\left(t_{i}\right)$ then yields a loop on $H\left(t_{i}\right) \backslash N(F)$ bounding a compressing disk in $W\left(t_{i}\right)$. This implies that there is a loop on $H\left(t_{i}-\epsilon\right) \backslash N(F)$ that bounds a compressing disk for $H\left(t_{i}-\epsilon\right)$ in $W\left(t_{i}-\epsilon\right)$. This violates the fact that the subinterval $\left(t_{i-1}, t_{i}\right)$ does not have the label $\mathbb{W}$.

Now let $\Gamma_{u}$ denote the union of the inessential loops of $H\left(t_{i}\right) \cap F$ and $\Gamma_{e}$ the union of the essential loops. The intersection set $H\left(t_{i}\right) \cap F$ thus consists of $\Gamma_{u}, \Gamma_{e}$, and a figure eight curve $C$. Let $N_{H}(C)$ denote a closed neighborhood of $C$ on $H\left(t_{i}\right)$. If some component $\alpha$ of $\partial N_{H}(C)$ bounds a disk in $H\left(t_{i}\right)$ that contains $C$ then we say $C$ was inessential.

Let $\pi: H \times I \rightarrow H$ denote projection onto the first factor. Let $\pi_{H}=\pi \circ \Phi^{-1}$. Then, for each $t \in(0,1)$, the function $\pi_{H} \mid H(t)$ is a map from $H(t)$ to $H$.

The sets $\pi_{H}\left(\Gamma_{u}\right)$ and $\pi_{H}\left(\Gamma_{e}\right)$ are isotopic to subsets of $\pi_{H}\left(H\left(t_{i}-\epsilon\right) \cap F\right)$ and $\pi_{H}\left(H\left(t_{i}+\epsilon\right) \cap F\right)$, for sufficiently small $\epsilon$. Such an isotopy induces an identification of $\Gamma_{u}$ and $\Gamma_{e}$ with subsets of $H\left(t_{i}-\epsilon\right) \cap F$ and $H\left(t_{i}+\epsilon\right) \cap F$. Furthermore the loop $\alpha$ (if it exists) can be identified with loops on $H\left(t_{i}-\epsilon\right)$ and $H\left(t_{i}+\epsilon\right)$ which are disjoint from $F$.

Let $H_{0}, H_{-}$and $H_{+}$denote the surfaces obtained by isotoping $H\left(t_{i}\right), H\left(t_{i}-\epsilon\right)$ and $H\left(t_{i}+\epsilon\right)$, preserving $\Gamma_{e}$, but removing $\Gamma_{u}$. In each case these isotopies can be achieved via a series of identical moves on innermost disks. Note that if the figure eight $C$ is inessential and surrounded by some loop of $\Gamma_{u}$ then it will disappear in the course of these isotopies.

Now suppose $C$ was inessential but did not disappear (and is therefore not surrounded by some loop of $\Gamma_{u}$ ). By definition $\alpha$ bounds a disk $D$ on $H_{0}$ (which can be identified with disks on $H_{-}$and $H_{+}$). As $F$ is incompressible any intersection of $D$ with $F$ can be removed by a further isotopy of $H_{0}, H_{-}$and $H_{+}$. Henceforth, we will assume that if $C$ is inessential then $\alpha$ bounds disks in $H_{0}, H_{-}$and $H_{+}$which are disjoint from $F$.

Let $V_{0}, W_{0}, V_{-}, W_{-}, V_{+}$, and $W_{+}$be the corresponding compression bodies bounded by $H_{0}, H_{-}$, and $H_{+}$. By assumption the interval $\left(t_{i-1}, t_{i}\right)$ does not have the label $\mathbb{W}$. It thus follows that no essential loop of $H_{-}$, disjoint from $F$, bounds a compressing disk in $W_{-}$. This is because only inessential loops are effected in the passage from $H\left(t_{i}-\epsilon\right)$ to $H_{-}$. Similarly we may conclude that no essential loop of $H_{+}$, disjoint from $F$, bounds a compressing disk in $V_{+}$. 
Assume, to obtain a contradiction, that $E^{\prime}$ is a compressing disk for a component $H^{\prime}$ of $H_{0} \backslash N(F)$. Since every loop of $H_{0} \cap F$ is essential on $H_{0}$, and $C$ was removed if it was inessential, it follows that $\partial E^{\prime}$ is essential on $H_{0}$. Furthermore, as only the inessential intersection curves were effected in the passage from $H\left(t_{i}\right)$ to $H_{0}$ it follows that $\partial E^{\prime}$ is an essential loop on $H\left(t_{i}\right)$, and is disjoint from $F$. It follows from Lemma 3.1 that there is a compressing disk $E$ for $H\left(t_{i}\right)$ with $\partial E=\partial E^{\prime}$. Hence $\partial E$ is also disjoint from $F$.

The loop $\partial E$ can be identified with essential loops of both $H\left(t_{i}-\epsilon\right) \backslash N(F)$ and $H\left(t_{i}+\epsilon\right) \backslash N(F)$ which bound similar compressing disks. We conclude the disk $E$ may be identified with a compressing disk for both $H_{-}$and $H_{+}$with boundary disjoint from $F$. If $E \subset W\left(t_{i}\right)$ then this violates the fact that there is no compressing disk for $H_{-}$in $W_{-}$with boundary disjoint from $F$. On the other hand, if $E \subset V\left(t_{i}\right)$, then we contradict the fact that there is no compressing disk for $H_{+}$in $V_{+}$with boundary disjoint from $F$.

We conclude that the components of $H_{0} \backslash N(F)$ are incompressible in the respective submanifolds of $M \backslash N(F)$, as asserted by the third conclusion of the lemma.

The third conclusion of Lemma 3.3 follows. This completes the proof of Lemma 3.3.

We now use the above result to establish the following lemma.

Lemma 3.11 Let $M$ be a compact, irreducible, orientable 3-manifold with $\partial M$ incompressible, if non-empty. Suppose $M=X \cup_{F} Y$, where $F$ is essential, connected, and closed. Suppose $M=V \cup_{H} W$, where $H$ is a Heegaard surface. Then either $H$ is an amalgamation of splittings of $X$ and $Y$ or there are properly embedded surfaces $H_{X} \subset X$ and $H_{Y} \subset Y$ with boundaries on $F$ such that at least one of the following holds:

(1) The surfaces $H_{X}$ and $H_{Y}$ are incompressible, not boundary parallel, $\partial H_{X}=$ $\partial H_{Y}$ and $\chi\left(H_{X}\right)+\chi\left(H_{Y}\right) \geq \chi(H)$.

(2) After possibly exchanging $X$ and $Y$ the surface $H_{X}$ is incompressible, not boundary parallel, the surface $H_{Y}$ is strongly irreducible, $\partial H_{X}=\partial H_{Y}$ and $\chi\left(H_{X}\right)+\chi\left(H_{Y}\right) \geq \chi(H)$.

(3) The surfaces $H_{X}$ and $H_{Y}$ are incompressible, not boundary parallel, $\partial H_{X} \cap$ $\partial H_{Y}=\varnothing$, and $\chi\left(H_{X}\right)+\chi\left(H_{Y}\right)-1 \geq \chi(H)$.

Remark 3.12 If $H$ is assumed to be strongly irreducible then we will show that each of the above inequalities can be replaced by equalities. 
Proof By Scharlemann-Thompson [12] we may untelescope the Heegaard splitting $H$. That is, there is a generalized Heegaard splitting $\left\{H_{i}\right\}_{i=0}^{2 n}$ of $M$ with thick and thin levels obtained from $H$ by some number of compressions. Furthermore, we can find such a generalized Heegaard splitting such that each thick level $H_{i}$ is strongly irreducible in the submanifold of $M$ cobounded by $H_{i-1}$ and $H_{i+1}$. It is shown in [12] that in such a generalized Heegaard splitting each thin level is incompressible in $M$.

Isotope $F$ to meet the set of thin levels of $\left\{H_{i}\right\}$ in a minimal number of curves. Suppose first that for some $i$, the surface $F$ is parallel to a component of the thin level $H_{2 i}$. Then the components of $\left\{H_{i}\right\}$ which meet $X$ form an untelescoped Heegaard splitting of $X$, and the components which meet $Y$ form an untelescoped Heegaard splitting of $Y$. Telescoping (the operation which is the inverse of untelescoping) now produces Heegaard splittings of $X$ and $Y$ with amalgamation $H$. Hence, the first conclusion of Lemma 3.11 follows.

Now suppose $F$ intersects the thin level $H_{2 i}$. Then $F$ divides $H_{2 i}$ into subsurfaces $H_{X} \subset X$ and $H_{Y} \subset Y$. We claim that $H_{X}$ is incompressible in $X$ and $H_{Y}$ is incompressible in $Y$. If not, then there is some compressing disk $D$ for $H_{X}$ (say) in $X$. As $H_{2 i}$ is incompressible in $M, \partial D$ bounds a disk $E$ in $H_{2 i}$. Since $\partial D$ is essential in $H_{X}$ but inessential in $H_{2 i}$ the surface $F$ must intersect the disk $E \subset H_{2 i}$. As $M$ is irreducible we can now do a sequence of isotopies to remove all curves of $E \cap F$, reducing the number of times $F$ meets the set of thin levels.

Since $F$ meets all thin levels minimally it also follows that neither $H_{X}$ nor $H_{Y}$ are boundary parallel. Finally, since $H_{2 i}=H_{X} \cup H_{Y}$, and $H_{2 i}$ is obtained from $H$ be some number of compressions, we have $\chi\left(H_{X}\right)+\chi\left(H_{Y}\right) \geq \chi(H)$. Hence, Case (1) of the conclusion of Lemma 3.11 follows.

We are now reduced to the case where $F$ misses all thin levels, and is parallel to none. Hence, $F$ is completely contained in a submanifold with incompressible boundary which has a strongly irreducible Heegaard splitting, obtained from $H$ by some number of compressions. It suffices, then, to prove Lemma 3.11 in the case where $H$ is strongly irreducible.

Use Lemma 3.3 to isotope $H$ so that it is transverse or almost transverse to $F$, and so that the conclusion of Lemma 3.3 follows. If $H$ is transverse to $F$ then let $H_{X}=H \cap X$ and $H_{Y}=H \cap Y$, and Case (1) or (2) of the lemma at hand follows.

The remaining case is when $H$ meets $F$ almost transversally. Let $p$ denote the saddle point of $H \cap F$. Isotope $H$ by pushing the point $p$ slightly into $Y$, to obtain the surface $H^{\prime}$. Hence, $H^{\prime}$ is transverse to $F$. Furthermore, any compressing disk for 
$H_{X}=H^{\prime} \cap X$ is a compressing disk for $H \cap X$, so there must be none by Lemma 3.3. We conclude $H_{X}$ is a properly embedded, incompressible surface in $X$. Similarly, by pushing $p$ slightly into $X$ we may obtain from $H$ a properly embedded, incompressible surface $H_{Y} \subset Y$.
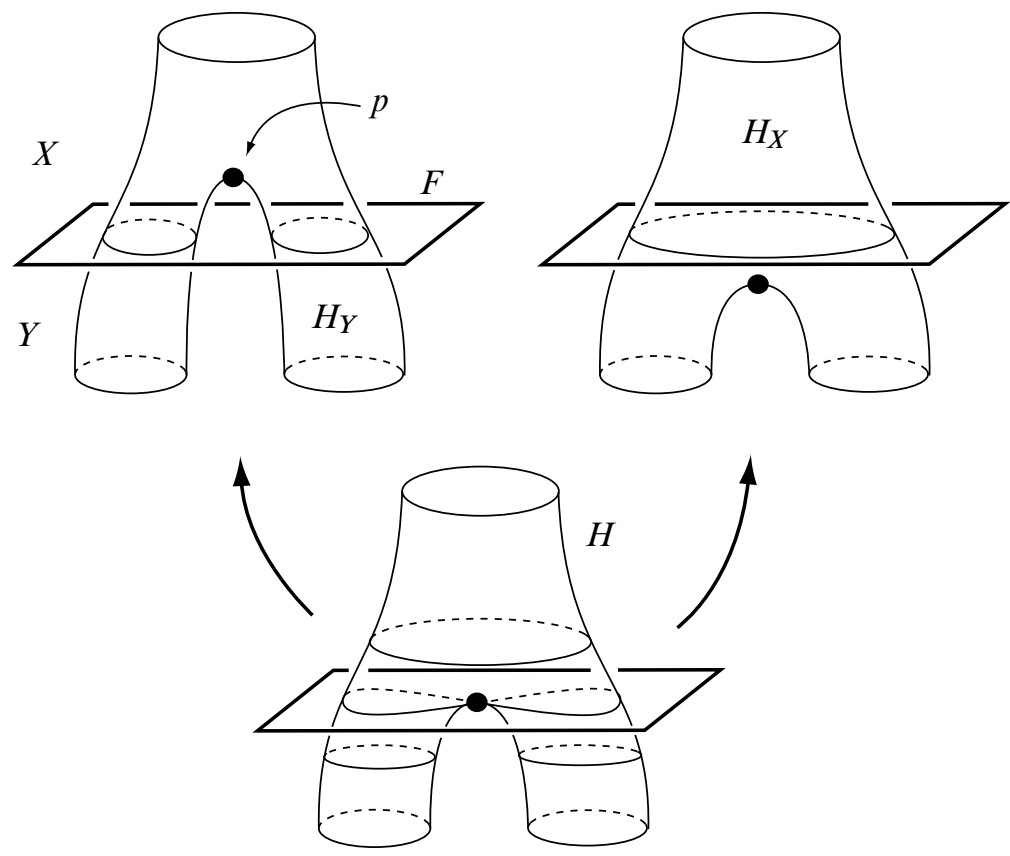

Figure 4: $H$ differs from $H_{X} \cup H_{Y}$ by a pair of pants.

As $H$ and $F$ are orientable, it follows that $H_{X} \cap F$ may be made disjoint from $H_{Y} \cap F$. Furthermore, the only essential difference between $H_{X} \cup H_{Y}$ and $H$ is a pair of pants, having Euler characteristic negative one (see Figure 4). Hence, Case (3) of the conclusion of Lemma 3.11 now follows.

\section{Manifolds with no strongly irreducible Heegaard splittings}

A knot manifold is a compact, orientable, irreducible three-manifold with a single boundary component, which is incompressible and homeomorphic to a torus. The goal of this section is to prove the following theorem:

Theorem 4.1 Suppose that $X$ and $Y$ are knot manifolds and $\varphi: \partial X \rightarrow \partial Y$ is a sufficiently complicated homeomorphism. Then the manifold $M(\varphi)=X \cup_{\varphi} Y$ has no strongly irreducible Heegaard splittings. 
Note the similarity of Theorem 4.1 to Cooper and Scharlemann's result [2]. That paper proves that if a 3-manifold is constructed by identifying the boundary components of $T^{2} \times I$ via a "sufficiently complicated" map then there are no strongly irreducible Heegaard splitting of the resulting 3-manifold.

To make the statement of Theorem 4.1 precise we must give a reasonable definition of the term sufficiently complicated. To this end fix, once and for all, psuedo-triangulations of $X$ and $Y$ with one vertex. (A psuedo-triangulation is a decomposition into simplices where any two such simplices intersect in a collection of lower dimensional simplices.) Let $\Delta(X)$ be the set of slopes in $\partial X$ which are the boundary of some normal or almost normal surface in $X$. Note that $\Delta(X)$ is finite, by a result of Jaco and Sedgwick [6] (see also Theorem 9.7 of Bachman [1] for a discussion of the almost normal case). Define $\Delta(Y)$ similarly.

Recall now the definition of the Farey graph, $\mathcal{F}(X)$. The vertices of $\mathcal{F}(X)$ are all slopes in $\partial X$. Two slopes are connected by an edge if they intersect once. The distance between two slopes is then defined to be the minimal number of edges required in a path connecting them. The distance between two sets of slopes is the minimal distance between their elements.

Definition 4.2 A map $\varphi: \partial X \rightarrow \partial Y$ is sufficiently complicated if the distance between $\Delta(X)$ to $\varphi^{-1}(\Delta(Y))$ inside of $\mathcal{F}(X)$ is at least two.

Remark 4.3 Note that, as $\Delta(X)$ and $\Delta(Y)$ are finite, "most" elements of $\mathcal{M C G}\left(\mathbb{T}^{2}\right)$ $\cong S L(2, \mathbb{Z})$ are sufficiently complicated, in the above sense. In particular any sufficiently large power of an Anosov map is sufficiently complicated. The same holds for all but a finite number of Dehn twists.

Before giving the proof of Theorem 4.1 we must discuss boundary compressions. Suppose $G \subset N$ is a properly embedded, two-sided surface in a compact, orientable, irreducible three-manifold $N$. We suppose further that $\partial N$ is incompressible in $N$. Suppose $D \subset N$ is a boundary compression for $G$.

Definition 4.4 The boundary compression $D$ is honest if $D \cap \partial N$ is essential as a properly embedded arc in $\partial N \backslash \partial G$. If $D$ is not honest it is dishonest.

Definition 4.5 Let $N$ be a knot manifold. We now define the banding, $\widehat{D}$, of a boundary compression $D$ for $G$. First assume $D$ is honest. Then $D \cap \partial N$ meets distinct boundary components of $\partial G$, as $G$ is orientable. These components of $\partial G$ cobound an annulus $A \subset \partial N$ such that $D \cap \partial N \subset A$. Let $D^{\prime}$ denote the disk obtained 
from $A$ by removing a neighborhood of $D \cap \partial N$ and attaching two parallel copies of $D$. Isotope $D^{\prime}$ to be disjoint from $\partial N$ while maintaining $\partial D^{\prime} \subset G$. The resulting disk is the desired banding $\widehat{D}$ of $D$.

Now suppose $D$ is dishonest. Then the arc $D \cap \partial N$ cobounds, with a subarc of $\partial G$, a subdisk $D^{\prime}$ of $\partial N$. The disk $\widehat{D}$ is obtained by pushing $D^{\prime \prime}=D \cup D^{\prime}$ into the interior of $N$, while maintaining $\partial D^{\prime \prime} \subset G$.

Note that when $C$ is a compressing disk and $D$ is a boundary-compressing disk (honest or dishonest) if $C \cap D=\varnothing$ then $C \cap \widehat{D}=\varnothing$.

Lemma 4.6 If $D$ is a boundary compression for $G$ and $\partial N=\mathbb{T}^{2}$ then $G$ is either compressible or the component of $G$ meeting $D$ is a boundary parallel annulus.

Recall that by a strongly irreducible surface we mean a properly embedded, twosided surface which compresses on both sides and all pairs of compressing disks on opposite sides must meet. We now strengthen this definition to account for boundary compressions, as in Bachman [1].

Definition 4.7 A properly embedded, separating surface is $\partial$-strongly irreducible if

(1) every compressing and boundary-compressing disk on one side meets every compressing and boundary-compressing disk on the other side, and

(2) there is at least one compressing or boundary-compressing disk on each side.

Lemma 4.8 Let $N$ be a knot manifold. Let $G$ be a separating, properly embedded, connected surface in $N$ which is strongly irreducible, has non-empty boundary, and is not peripheral. Then either $G$ is $\partial$-strongly irreducible or $\partial G$ is at most distance one from the boundary of some properly embedded surface which is both incompressible and boundary-incompressible.

Proof Suppose $G$ divides $N$ into $V$ and $W$. If $G$ is not $\partial$-strongly irreducible then there are disjoint disks $D \subset V$ and $E \subset W$ such that at least one, say $D$, is a boundary-compressing disk. The disk $E$ is either a compression or a boundary compression.

Since $G$ is not a boundary parallel annulus we know by Lemma 4.6 that the banding disk $\widehat{D}$ is a compressing disk for $G$. If $E$ is a compressing disk then $E \cap D=\varnothing$ implies that $E \cap \widehat{D}=\varnothing$, contradicting strong irreducibility. We conclude $E$ is a boundary compression. 
Let $G^{\prime}$ denote the result of boundary-compressing $G$ along both $D$ and $E$. Let $V^{\prime}$ and $W^{\prime}$ denote the sides of $G^{\prime}$ which correspond to $V$ and $W$. We now claim that $G^{\prime}$ is incompressible. Suppose $D^{\prime}$ is a compressing disk for $G^{\prime}$ in $V^{\prime}$. Then $D^{\prime}$ must have been a compressing disk for $G$ in $V$ which was disjoint from $E$, and hence disjoint from $\widehat{E}$. This contradicts the strong irreducibility of $G$. By symmetry we conclude $G^{\prime}$ is incompressible.

We now claim $G^{\prime}$ is boundary incompressible as well. Suppose $C$ is a boundarycompressing disk for $G^{\prime}$. Since $G^{\prime}$ is incompressible we know $\widehat{C}$ is not a compressing disk, so it follows from Lemma 4.6 that $G^{\prime}$ must be a boundary parallel annulus. It follows that all of $G$ was isotopic into a neighborhood of $\partial N$, contradicting our hypotheses.

It remains only to show that $\partial G$ is at a distance of at most one from $\partial G^{\prime}$. In order for the slope of $\partial G^{\prime}$ to be different from the slope of $\partial G$ all of the loops of $\partial G$ must meet either $D$ or $E$. This immediately implies $|\partial G| \leq 4$. The possibility that $|\partial G|$ is one or three is ruled out by the fact that $G$ is separating. The fact that $D$ and $E$ are on opposite sides of $G$ rules out $|\partial G|=4$, since we are assuming that every component of $\partial G$ meets either $D$ or $E$.

If $|\partial G|=2$, both $D$ and $E$ are dishonest, and each meets different components of $\partial G$ then $\widehat{D} \cap \widehat{E}=\varnothing$. This violates the strong irreducibility of $G$.

There are three remaining cases. In each of these cases $|\partial G|=2$ and both boundary loops are affected by the transition to $G^{\prime}$. See Figure 5. In the top picture both $D$ and $E$ are honest. The two loops of $\partial G$ are transformed into two loops, both distance one from the original. In the middle picture exactly one of the disks $D$ or $E$ is dishonest, and the boundary slope remains unchanged. The configuration depicted at the bottom of Figure 5 cannot happen, since it represents a situation in which $\widehat{D}$ is disjoint from $\widehat{E}$, contradicting the strong irreducibility of $G$.

We conclude with:

Proof of Theorem 4.1 Suppose that $X$ and $Y$ are triangulated knot manifolds, as above. Fix a gluing $\varphi: \partial X \rightarrow \partial Y$. Suppose that $H \subset M(\varphi)=X \cup_{\varphi} Y$ is a strongly irreducible Heegaard splitting surface. Let $F \cong \mathbb{T}^{2}$ be the image of $\partial X$ inside of $M(\varphi)$.

Now apply Lemma 3.3 and Remark 3.4 to the pair $H$ and $F$ in $M(\varphi)$. Let $H_{X}$ be a component of $H \cap X$ which is incompressible and not a boundary parallel annulus, if such exists. If no such component exists take $H_{X}$ to be the non-boundary parallel component of $H \cap X$. In this case $H_{X}$ is strongly irreducible. (At least one component 

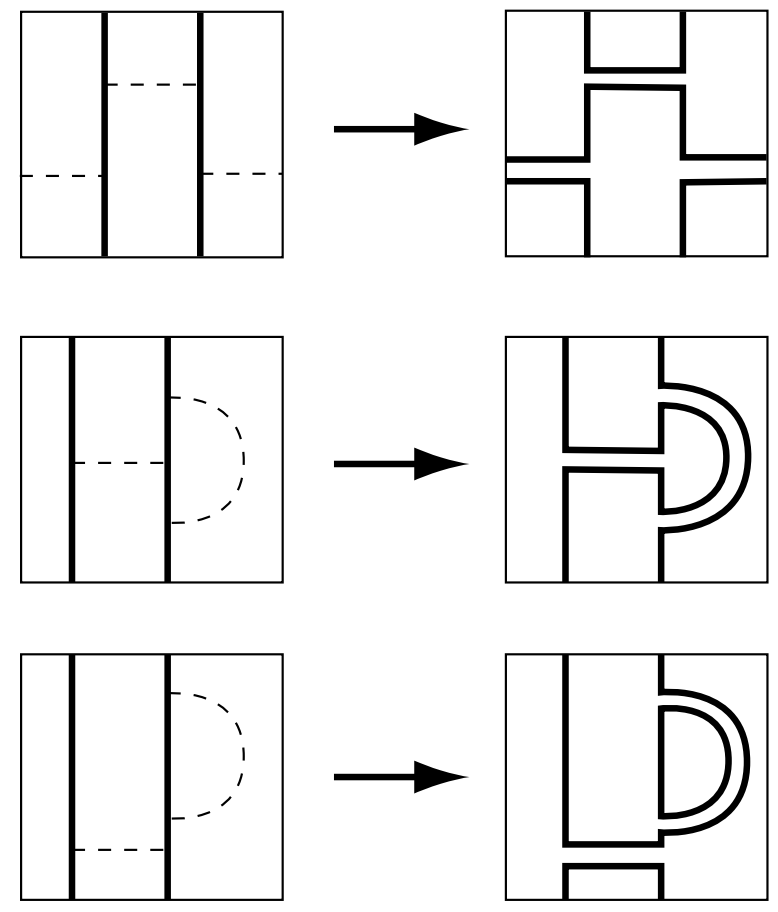

Figure 5: Possible effects of boundary-compression on $\partial G$

of $H \cap X$ is not boundary parallel. Otherwise $H$ is isotopic into $Y$, a contradiction.) Choose $H_{Y}$ similarly and note that, by Lemma 3.3, not both of $H_{X}$ and $H_{Y}$ are strongly irreducible. Note that $\partial H_{X}$ and $\varphi^{-1}\left(\partial H_{Y}\right)$ have the same slope.

Suppose that $H_{X}$ and $H_{Y}$ are both incompressible. As $\partial X \cong \partial Y \cong \mathbb{T}^{2}$ it follows from Lemma 4.6 that $H_{X}$ and $H_{Y}$ are also boundary incompressible. So $H_{X}$ and $H_{Y}$ may be normalized with respect to the given triangulations Haken [3]. It follows that the sets $\Delta(X)$ and $\varphi^{-1}(\Delta(Y))$ intersect and thus $\varphi$ is not sufficiently complicated.

Suppose now that $H_{X}$ is incompressible and thus boundary incompressible. Suppose that $H_{Y}$ is a strongly irreducible surface. Then, by Lemma 4.8, either $H_{Y}$ is $\partial-$ strongly irreducible or $\partial H_{Y}$ intersects the boundary of some incompressible, boundary incompressible surface $H_{Y}^{\prime}$ at most once. In the latter case $H_{Y}^{\prime}$ may be normalized, and hence $\partial H_{Y}^{\prime} \in \Delta(Y)$. In the former case it follows from work of the first author (Corollary 8.9 of [1]) that the surface $H_{Y}$ is properly isotopic to an almost normal surface, and so $\partial H_{Y} \in \Delta(Y)$. In either case we see $\partial H_{X}$ (an element of $\Delta(X)$ ) is within distance one from some element of $\varphi^{-1}(\Delta(Y))$ and hence $\varphi$ is not sufficiently complicated. 


\section{Amalgamating small manifolds}

Let $X$ be a manifold with boundary. The tunnel number of $X, t(X)$ is the minimal number of properly embedded arcs that need to be drilled out of $X$ to obtain a handlebody; i.e. so that $X \backslash N(\operatorname{arcs})$ is a handlebody. The handle number of $X$ is the minimal number of properly embedded arcs that need to be drilled out of $X$ to obtain a compression body; i.e. so that $X \backslash N(\operatorname{arcs})$ is a compression body. If $|\partial X|=1$ then $t(X)=h(X)$.

Let $M=X \cup_{F} Y$ be a manifold obtained by gluing $X$ and $Y$, two connected small manifolds with incompressible boundary, along a collection of boundary components homeomorphic to a surface $F$. The goal of this section is to show that the Heegaard genera of $X$ and $Y$ are bounded in terms of the Heegaard genus of $M=X \cup_{F} Y$. More specifically, we establish:

Theorem 5.1 Let $M$ be a compact, orientable 3-manifold with incompressible boundary. Suppose $M$ is obtained by gluing two connected, small manifolds along a union of incompressible boundary components, $M=X \cup_{F} Y$. Then the following statements hold:

(1) $g(M) \geq \frac{1}{2}(h(X)+h(Y))$

(2) if $M$ is closed and $F$ is connected, $g(M) \geq \frac{1}{2}(t(X)+t(Y))$

(3) $g(M) \geq \frac{1}{2}(g(X)+g(Y)-2 g(F))$.

The theorem is motivated by the fact that a properly embedded, incompressible surface cuts a small manifold into one or two compression bodies.

We begin with the following definitions. Let $F$ be an orientable surface, possibly with boundary components, and possibly disconnected. Let $C$ be the manifold obtained by forming $F \times I$ and attaching one handles to the surface $F \times\{1\}$. Then $C$ is a relative compression body. We label the boundary as follows: the negative boundary is $\partial_{-} C=F \times\{0\}$, the vertical boundary is $\partial_{V} C=\partial F \times I$, and the positive boundary is $\partial_{+} C=\overline{\partial C \backslash\left(\partial_{-} C \cup \partial_{V} C\right)}$. The vertical boundary is a collection of annuli. It is important to note that a given manifold may admit many relative compression body structures. For example, if $F$ is a surface with boundary and $C=F \times I$, then $C$ can be thought of as a relative compression body with $\partial_{-} C=F \times\{0\}$, or $C$ can be thought of as a handlebody with $\partial_{-} C=\varnothing$. In fact, given a relative compression body $C$, it is always possible to think of $C$ as a (non-relative) compression body by promoting all non-closed components of $\partial_{-} C$ and all components of $\partial_{V} C$ to the positive boundary. 
A relative Heegaard splitting is the union of two relative compression bodies, identified along their positive boundaries. The splitting will be considered non-trivial if neither relative compression body is a product; i.e. both compression bodies have 1-handles.

Lemma 5.2 Let $X$ be a manifold that admits a non-trivial, strongly irreducible and relative Heegaard splitting $X=C_{1} \cup C_{2}$. Then $\partial_{-} C_{1}$ and $\partial_{-} C_{2}$ are incompressible in $X$.

Proof An examination of the proof of the Haken Lemma [4] (see also Jaco [5]) will reveal that it applies directly to the case of relative Heegaard splittings. In particular, if either $\partial_{-} C_{1}$ or $\partial_{-} C_{2}$ has compressible boundary, then there is a compressing disk $D$ for the boundary component that meets the splitting surface in a single closed loop. The loop decomposes the compressing disk into a vertical annulus in one compression body, say $C_{1}$, and a disk $D_{2} \subset C_{2}$. Since $C_{1}$ is not a product we can find a compressing disk $D_{1}$ for $\partial_{+} C_{1}$, disjoint from the annulus, and hence disjoint from $D_{2}$. The pair $\left(D_{1}, D_{2}\right)$ contradicts strong irreducibility of the relative Heegaard splitting.

Lemma 5.3 An irreducible connected small manifold with compressible boundary is a compression body.

Proof Let $X$ be a connected small manifold with compressible boundary. In an optimistic fashion, denote a compressible boundary component by $\partial_{+} X$ and all other components by $\partial_{-} X$. Since $\partial_{+} X$ is compressible it bounds a (not properly embedded) submanifold $C$ of $X$ which is a compression body, so that $\partial_{+} C=\partial_{+} X$. Choose $C$ to be maximal in this regard. Precisely, choose $C$ so that $\partial_{-} C$ contains no $2-$ spheres ( $X$ is irreducible) and so that $\sum\left(1-\chi\left(S_{i}\right)\right)$ is minimal, where $\left\{S_{i}\right\}$ are the components of $\partial_{-} C$.

If $S$ is a component of $\partial_{-} C$ then $S$ is incompressible in $C$. Suppose $D$ is a compressing disk for $S$ in $X \backslash C$. Then $D$ is the core of a 2-handle that we can attach to $C$ to obtain a new compression body with negative boundary "smaller" than that of $C$. This contradicts our minimality assumption. We conclude $S$ is incompressible in $X \backslash C$. As $X$ is small, $S$ must be peripheral, and since $C$ is not a product, it is parallel in $X \backslash C$ to a component of $\partial_{-} X$. The (possibly disconnected) surface $\partial_{-} C$ separates the components of $\partial_{-} X$ from $\partial_{+} X$, so each component of $\partial_{-} X$ is in fact parallel to a component of $\partial_{-} C$. The parallelism yields an isotopy between $X$ and $C$. $X$ is therefore a compression body. Note that only one boundary component, $\partial_{+} X$, is compressible. 
Theorem 5.4 Let $H_{X}$ be a non-peripheral, connected, incompressible surface that is properly embedded in a connected, small manifold $X$. Then $h(X) \leq 1-\chi\left(H_{X}\right)$. If $X$ has a single boundary component or $H_{X}$ meets every boundary component of $X$, then this applies to the tunnel number: $t(X) \leq 1-\chi\left(H_{X}\right)$.

Proof Let $\partial_{1} X$ denote those boundary components of $X$ that meet $H_{X}$ and $\partial_{2} X$ denote those boundary components which do not meet $H_{X}$.

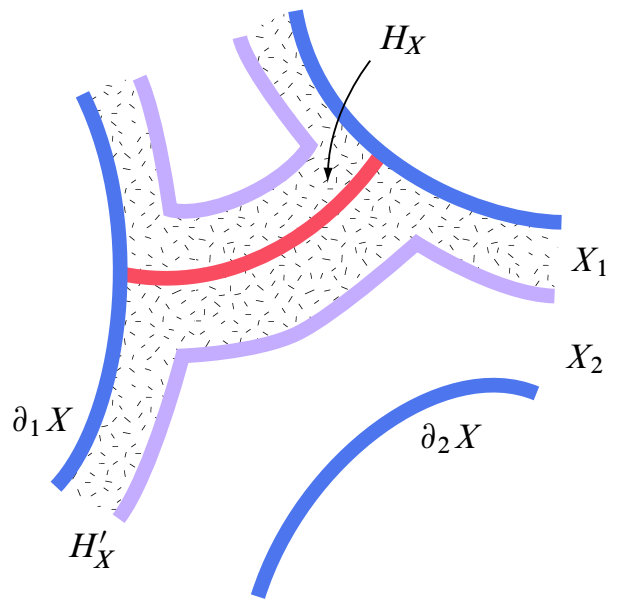

Figure 6: Labelling the boundary components of $X$

Let $X_{1}=\overline{N\left(H_{X} \cup \partial_{1} X\right)}$ and $X_{2}=\overline{X \backslash X_{1}}$. This decomposes $X$ into $X=X_{1} \cup_{H_{X}^{\prime}} X_{2}$, where $H_{X}^{\prime}$ is the common boundary of $X_{1}$ and $X_{2}$. See the schematic in Figure 6. Note that $\partial_{1} X$ and $H_{X}$ are contained in $X_{1}$ and $\partial_{2} X$ is contained in $X_{2}$. Since $H_{X}$ is connected it follows that $X_{1}$ is connected. If $H_{X}$ separates $X$ then $X_{2}$ will have two components.

The surface $H_{X}^{\prime}$ will have two components if $H_{X}$ separates and one component otherwise. Since $X$ is a small manifold, each component of $H_{X}^{\prime}$ is either compressible in $X$ or peripheral to a boundary component of $X$.

Claim If a component of $H_{X}^{\prime}$ is compressible, it is compressible into $X_{2}$.

Proof If there is a compressing disk for the compressible component of $H_{X}^{\prime}$ then there is one that is disjoint from $H_{X}$. This is because any intersection could be removed by surgery. If $H_{X}^{\prime}$ has two components then $H_{X}$ separates them. Hence our chosen compressing disk does not meet the other component of $H_{X}^{\prime}$. Therefore, our compressing disk is properly embedded in either $X_{1} \backslash N\left(H_{X}\right)$ or $X_{2}$. But, $X_{1} \backslash N\left(H_{X}\right)$ 
is a product and has incompressible boundary. It follows that a compressible component of $H_{X}^{\prime}$ is compressible into $X_{2}$.

Claim No component of $H_{X}^{\prime}$ is peripheral into $\partial_{1} X$.

Proof If this occurred, $X_{1}$ would be contained in a product neighborhood of a boundary component. This in turn implies that $H_{X}$ was peripheral.

Claim Each component of $X_{2}$ is a compression body.

Proof Suppose that a component $X^{\prime}$ of $X_{2}$ contains a closed non-peripheral essential surface $G$. Since $X$ is small, $G$ is either compressible in $X$ or parallel to a component $G^{\prime}$ of $\partial X \backslash \partial X^{\prime}$. In the latter case $G^{\prime} \subset \partial_{1} X$ or $G^{\prime} \subset \partial_{2} X \backslash \partial X^{\prime}$. If $G^{\prime} \subset \partial_{2} X \backslash \partial X^{\prime}$ then $H_{X}$ separates $G$ from $G^{\prime}$.

Since $H_{X}$ is incompressible, any compressing disk $D \subset X$ for $G$ can be isotoped so that it does not intersect $H_{X}$, and so can be isotoped to miss $X_{1}$. Therefore $G$ is compressible in $X_{2}$, contradicting the essentiality of $G$. If $G^{\prime} \subset \partial_{1} X$ or $G^{\prime} \subset$ $\partial_{2} X \backslash \partial X^{\prime}$ then there is a product containing $H_{X}$. In particular, this implies that $H_{X}$ is contained in a product neighborhood of $\partial X$, contradicting the fact that $H_{X}$ is not peripheral. Thus, $X_{2}$ is small.

Each component of $H_{X}^{\prime}$ is therefore compressible into $X_{2}$ or parallel to a component of $\partial_{2} X$. In either case, by Lemma 5.3 or by parallelism, $H_{X}^{\prime}=\partial_{+} X_{2}$, where $X_{2}$ is either one or two compression bodies.

It is now straightforward to build a handle system for $X$ (tunnel system in the case that $\left.\partial_{1} X=\partial X\right)$. Choose $\tau$, a minimal collection of arcs that are properly embedded in $H_{X}$ and that cut $H_{X}$ into a single disk $D$. The collection $\tau$ contains $1-\chi\left(H_{X}\right)$ arcs. Moreover, $\tau$ is a handle system that induces a Heegaard splitting, $X=C_{1} \cup C_{2}$, where $C_{1}=\overline{N\left(\partial_{1} X \cup \tau\right)}$ and $C_{2}=\overline{X \backslash C_{1}}$. Clearly $C_{1}$ is a compression body. $C_{2}$ is a compression body because it is formed by attaching a 1-handle (a neighborhood of the cocore of $D$ ) to the positive boundary of the compression body/bodies $X_{2}$. This completes the proof of Theorem 5.4.

Theorem 5.5 Let $H_{X}$ be a non-peripheral, bi-compressible, connected, strongly irreducible surface properly embedded in a connected, small manifold $X$. Then $h(X) \leq$ $1-\chi\left(H_{X}\right)$. If $X$ has a single boundary component, then this applies to the tunnel number: $t(X) \leq 1-\chi\left(H_{X}\right)$. 
Proof We may apply the previous theorem if $X$ also contains a non-peripheral incompressible surface with boundary whose negative Euler characteristic is less than that of $H_{X}$. We may therefore assume that $H_{X}$ is a separating surface; if not we may compress $H_{X}$ to obtain such an incompressible surface. As before we will let $\partial_{1} X$ denote those boundary components of $X$ that meet $H_{X}$ and $\partial_{2} X$ denote those boundary components which do not meet $H_{X}$.

By compressing $H_{X}$ maximally to both sides, we define a relative Heegaard splitting of a submanifold $X^{\prime}=C_{1} \cup_{H_{X}} C_{2} \subset X$. Since we have compressed maximally, the negative boundary components of $C_{1}$ and $C_{2}$ are incompressible outside $X^{\prime}$. They are incompressible inside $X^{\prime}$ by Lemma 5.2. If any component is non-peripheral, we have our conclusion via Theorem 5.4. Each component of $\partial_{-} C_{i}, i=1,2$ is therefore peripheral. It now follows from the fact that $H_{X}$ is non-peripheral that $X^{\prime}$ is isotopic to $X$.

As in the earlier theorem, this structure defines a handle system for $X$. Choose $\tau$, a minimal collection of arcs that are properly embedded in $H_{X}$ and that cut $H_{X}$ into a single disk $D$. Now, $\tau$ is a handle system for $X$ that induces the Heegaard splitting, $X=C_{1}^{\prime} \cup C_{2}^{\prime}$, where $C_{1}^{\prime}=\overline{N\left(\partial_{1} X \cup \tau\right)}$ and $C_{2}^{\prime}=\overline{X \backslash C_{1}^{\prime}}$. Clearly $C_{1}^{\prime}$ is a compression body. $C_{2}^{\prime}$ is a compression body because it can be obtained by first promoting the vertical and non-closed negative boundary components of $C_{1}$ and $C_{2}$ and then joining the positive boundary of these (non-relative) compression bodies with a 1-handle (a neighborhood of the cocore of $D$ ).

The handle number of $X$ is thus bounded by $1-\chi\left(H_{X}\right)$.

Proof of Theorem 5.1 Let $H$ be a minimal genus splitting of $M$. If $H$ is an amalgamation of splittings of $X$ and $Y$, then the result holds trivially. Otherwise, by Lemma 3.11 we can construct properly embedded non-boundary parallel surfaces $H_{X}^{\prime} \subset X$ and $H_{Y}^{\prime} \subset Y$ so that each is either incompressible or strongly irreducible. As neither surface is boundary-parallel they contain components $H_{X} \subset H_{X}^{\prime}$ and $H_{Y} \subset H_{Y}^{\prime}$ which are non-boundary parallel and either incompressible or strongly irreducible. Furthermore, $\chi\left(H_{X}\right)+\chi\left(H_{Y}\right) \geq \chi(H)=2-2 g(M)$, or equivalently, $g(M) \geq \frac{1}{2}\left(2-\chi\left(H_{X}\right)-\chi\left(H_{Y}\right)\right)$.

By either Theorem 5.4 or Theorem 5.5, $X$ and $Y$ admit handle systems that are attached to components of $F$ and so that the number of handles is at most $1-\chi\left(H_{X}\right)$ and $1-\chi\left(H_{Y}\right)$, respectively. The first two assertions of Theorem 5.1 follow.

Our induced splitting of $X$ is obtained by attaching $1-\chi\left(H_{X}\right)$ handles to $F$. The genus of $X$ is therefore bounded by

$$
g(X) \leq g(F)+1-\chi\left(H_{X}\right) .
$$


Since a symmetric bound holds for $g(Y)$ we obtained the third conclusion of Theorem 5.1.

\section{References}

[1] D Bachman, Heegaard splittings with boundary and almost normal surfaces, Topology Appl. 116 (2001) 153-184 MR1855961

[2] D Cooper, M Scharlemann, The structure of a solvmanifold's Heegaard splittings, Turkish J. Math. 23 (1999) 1-18 MR1701636

[3] W Haken, Theorie der Normalfächen, Acta Math. 105 (1961) 245-375 MR0141106

[4] W Haken, Some results on surfaces in 3-manifolds, from: "Studies in Modern Topology", Math. Assoc. Amer. (distributed by Prentice-Hall, Englewood Cliffs, N.J.) (1968) 39-98 MR0224071

[5] W Jaco, Lectures on three-manifold topology, CBMS Regional Conference Series in Mathematics 43, American Mathematical Society, Providence, R.I. (1980) MR565450

[6] W Jaco, E Sedgwick, Decision problems in the space of Dehn fillings, Topology 42 (2003) 845-906 MR1958532

[7] K Johannson, Topology and combinatorics of 3-manifolds, Lecture Notes in Mathematics 1599, Springer, Berlin (1995) MR1439249

[8] H Kneser, Geschlossene Flächen in driedimensionalen Mannigfaltigkeiten, Jahresbericht der Dent. Math. Verein 28 (1929) 248-260

[9] M Lackenby, The Heegaard genus of amalgamated 3-manifolds, Geom. Dedicata 109 (2004) 139-145 MR2113191

[10] JH Rubinstein, Polyhedral minimal surfaces, Heegaard splittings and decision problems for 3-dimensional manifolds, from: "Geometric topology (Athens, GA, 1993)”, AMS/IP Stud. Adv. Math. 2, Amer. Math. Soc., Providence, RI (1997) 1-20 MR1470718

[11] M Scharlemann, Local detection of strongly irreducible Heegaard splittings, Topology Appl. 90 (1998) 135-147 MR1648310

[12] M Scharlemann, A Thompson, Thin position for 3-manifolds, from: "Geometric topology (Haifa, 1992)", Contemp. Math. 164, Amer. Math. Soc., Providence, RI (1994) 231-238 MR1282766

[13] J Schultens, Heegaard genus formula for Haken manifolds MR1226608 arXiv: math.GT/0108028

[14] J Schultens, The classification of Heegaard splittings for (compact orientable surface) $\times S^{1}$, Proc. London Math. Soc. (3) 67 (1993) 425-448 MR1226608 
Mathematics Department, Pitzer College

1050 North Mills Avenue, Claremont CA 91711, USA

Department of Mathematics, Rutgers, The State University of New Jersey

110 Frelinghuysen Rd, Piscataway NJ 08854-8019, USA

CTI, DePaul University, $243 \mathrm{~S}$ Wabash Avenue

Chicago IL 60604, USA

bachman@pitzer.edu, saulsch@math.rutgers.edu, esedgwick@cs.depaul.edu

Received: 26 July 2005 Revised: 18 January 2006

Algebraic $\mathcal{B}$ Geometric Topology, Volume 6 (2006) 